\title{
Spatial separation of spectrum inside the tapered metamaterial optical waveguide
}

\author{
CHENG XiaoChao, FU QuanHong \& ZHAO XiaoPeng* \\ Smart Materials Laboratory, Department of Applied Physics, Northwestern Polytechnical University, Xi'an 710129, China
}

Received August 26, 2009; accepted January 19, 2010

\begin{abstract}
In this work, the optical metamaterials based on silver dendritic cells are prepared by electrochemical deposition, and its transmission and focusing behaviors are investigated. The experimental results show that the optical metamaterials reveal a multiple pass-band transmission spectrum and a prominent focusing effect at the wavelength corresponding to the maximum transmission coefficient. Two optical metamaterial samples are combined into a tapered optical waveguide, and the spectra of transmitted light at the surface of the tapered optical waveguide is measured by using the fiber spectrometer along the light propagation direction. The results demonstrate that each frequency component of the wave packet is stopped at a different waveguide thickness, leading to the spatial separation of its spectrum. The spatial separation of spectrum can be effectively tuned by adjusting the inclination of the tapered optical waveguide, which can be used for storing photons and slow-light research.
\end{abstract}

metamaterial, silver dendritic structure, tapered optical waveguide, spatial separation of spectrum

Citation: Cheng X C, Fu Q H, Zhao X P. Spatial separation of spectrum inside the tapered metamaterial optical waveguide. Chinese Sci Bull, 2011, 56: 209-214, doi: 10.1007/s11434-010-4344-4

A metamaterial is an artificially structured material which possesses exotic properties compared with the normal material in nature. In 1968, Veselago [1] investigated theoretically the electromagnetic properties of substances with simultaneously negative permittivity $\varepsilon$ and negative permeability $\mu$, which are also called left-handed metamaterials (LHMs). Since Shelby et al. [2] assembled the array of split resonator rings (SRRs) and metallic wires and firstly realized LHMs at microwave frequencies in 2001, the researches on LHMs have attracted a lot of interest. LHMs have plenty of peculiar electromagnetic properties, such as negative refractive index [3], inverse Cherenkov radiation [4] and perfect lens [5]. So far, LHMs have been achieved at microwave, THz, infrared and visible frequencies [6-11]. However, restricted by the model of periodically structured LHMs, the fabrication of LHMs at optical frequencies using the electron-beam lithography or focused ion-beam techniques was difficult. The top-down approach often needs

*Corresponding author (email: xpzhao@nwpu.edu.cn) expensive equipments and involves complicated processes. Moreover, the effective area of the prepared sample is only of the micron-square scale, which greatly restricts the research and application on LHMs at visible frequencies. In previous studies, our group designed a dendritic structure which could provide a negative refractive index [12,13] and studied the electromagnetic properties of LHMs with defects, demonstrating that the optical metamaterials composed of random dendritic cells possess a multi-band resonance and negative refractive index [14-16]. Based on the model of random dendritic cells structured LHMs, our group proposed a bottom-up approach of electrochemical deposition to fabricate LHMs. In 2008, Liu et al. [17] prepared LHMs at infrared frequencies. For the electrochemical deposition method is simple, economical and highly effective, it can easily obtain nanometer metal structure by controlling experimental conditions, opening up a new way for researches and the application of optical metamaterials.

In 2007, Hess et al. [18] demonstrated theoretically that an axially varying heterostructure with a metamaterial core 
of negative refractive index can be used to efficiently and coherently bring light to a complete standstill. Each frequency component of the wave packet is stopped at a different guide thickness, leading to the spatial separation of its spectrum and the formation of a 'trapped rainbow' [18]. The 'trapped rainbow' method provides a new approach for slow-light research and photon storage [19,20]. Restricted by the size of optical LHMs fabricated by the electron-beam lithography or focused ion-beam techniques, the 'trapped rainbow' effect cannot be experimentally realized. Based on previous study [21], in this paper, the silver dendritic optical metamaterials are prepared using the electrochemical deposition, and the transmission and the focusing behaviors are investigated. The experimental results show that the optical metamaterial samples reveal a multiple pass- band transmission spectrum and a prominent focusing effect at the peak wavelength corresponding to the maximum transmission coefficient. Two silver dendritic optical metamaterials with the operation frequency range from 600 to $1750 \mathrm{~nm}$ are combined into a tapered optical waveguide, and the optical response is studied by measuring the spectra of transmitted light at the surface of the tapered optical waveguide along the light propagation direction. The results demonstrate that each frequency component of the wave packet is stopped at a different waveguide thickness, leading to the spatial separation of its spectrum. The spatial separation of spectrum can be effectively tuned by adjusting the inclination of the tapered waveguide, which can be used for storing photons and slow-light investigation, opening a way for the application of optoelectronic devices in "quantum information" processing, communication networks, and signal processing.

\section{Preparation of silver dendritic metamaterials and investigation of its optical properties}

\subsection{The preparation of silver dendritic optical meta- materials}

The silver dendritic cells are fabricated on two pieces of ITO glass $(55 \mathrm{~mm} \times 9 \mathrm{~mm})$ using the electrochemical deposition method. The optimum technical conditions are as follows: suitable mass fraction of silver nitrate solution is 8.35\%; the anode and the cathode are flat silver plate (purity 99.9\%) and ITO glass, respectively; the electrode gap is $0.625 \mathrm{~mm}$. In order to obtain silver dendritic cells with different sizes and densities on ITO glasses, which could possess metamaterial behaviors at different frequencies, the deposition voltage is adjusted from 0.4 to $0.8 \mathrm{~V}$, and the deposition time is adjusted from $80 \mathrm{~s}$ to $100 \mathrm{~s}$. The effective area of fabricated metamaterial samples is about $30 \mathrm{~mm} \times 9$ $\mathrm{mm}$. Afterwards, a PVA film with a nanometer-scale thickness was prepared as a dielectric spacer on the silver dendritic cells on one of the two ITO glasses. Then, the two ITO glasses were tightly combined with the silver dendritic cells face to face to form an optical metamaterial. The preparation conditions of PVA solution are as follows: (1) pour $15 \mathrm{~g}$ PVA and $500 \mathrm{~mL}$ ultrapure water into a beaker; (2) stir and heat until boiling; (3) transfer the PVA aqueous solution to a $500 \mathrm{~mL}$ volumetric flask after the PVA is dissolved and add ultrapure water until $500 \mathrm{~mL}$. Finally, the PVA aqueous solution ( $3 \mathrm{wt} \%$ ) is prepared.

Figure 1 shows the silver dendritic cells under different deposition voltages and times. The deposition voltages in (a), (b), (c) and (d) are 0.4, 0.6, 0.8, and $2.0 \mathrm{~V}$, respectively, and the deposition times are 80, 90, 100 and $100 \mathrm{~s}$, respectively. From Figure 1 we can see that the size of silver dendritic cells will be smaller and the density will be higher with higher deposition voltage and longer deposition time. Figure 1(a), (b) and (c) show that the silver dendritic cells have multi-level structures under optimal voltage from 0.4 to $0.8 \mathrm{~V}$. When the deposition voltage increases to $2.0 \mathrm{~V}$, however, the silver dendritic cells vanish and the silver particles appear instead, as shown in Figure 1(d).

\subsection{Results and discussion of the optical properties}

The transmission spectra of the metamaterial samples are measured by U-4100 spectrophotometer; the slab focusing experiments [22,23] are performed using a monochromator, a fiber spectrometer and a micron motorized positioning systems.

Figure 2 shows the transmission spectra of the samples in the wavelength range from 600 to $1750 \mathrm{~nm}$. Curves $\mathrm{a}, \mathrm{b}$ and $\mathrm{c}$ are the transmission spectra of ITO/PVA/ITO (I/P/I), ITO/silver dendritic structures/PVA/silver dendritic structures/ITO (I/d/P/d/I) and ITO/silver film structures/PVA/ silver film structures/ITO (I/f/P/f/I). By comparing curves $\mathrm{a}, \mathrm{b}$ and $\mathrm{c}$, we can see that only the transmission spectrum of the sample with silver dendritic structures reveals multiple pass-bands. Based on previous studies of our group, it is believed that the multiple pass-bands of the spectrum are the result of the different size of the silver dendritic cells. Similarly, the magnetic resonance frequency of the silver dendritic structure is closely related to its structural parameters for split ring resonators. The silver dendritic cells of a certain size cause a resonance at a certain frequency. In the tested region of the samples, the size of the dendritic unit cells is non-uniform, so it gives multiple resonances and reveals multiple transmission peaks. If the size of the unit cells is highly uniform, there will be only one transmission peak which appears at the resonance frequency. As reported previously, the dendritic unit cells of uniform size have been used and there was only one transmission peak.

The results of the slab focusing experiment are shown in Figure 3. It could be found that the light intensity of transmission spectra of the dendritic sample increases by $32 \%$, $22 \%, 21.6 \%, 51.4 \%, 20.85 \%$ and $62.2 \%$, at the related wavelength of transmission peak of $645 \mathrm{~nm}, 708 \mathrm{~nm}, 788 \mathrm{~nm}$, 

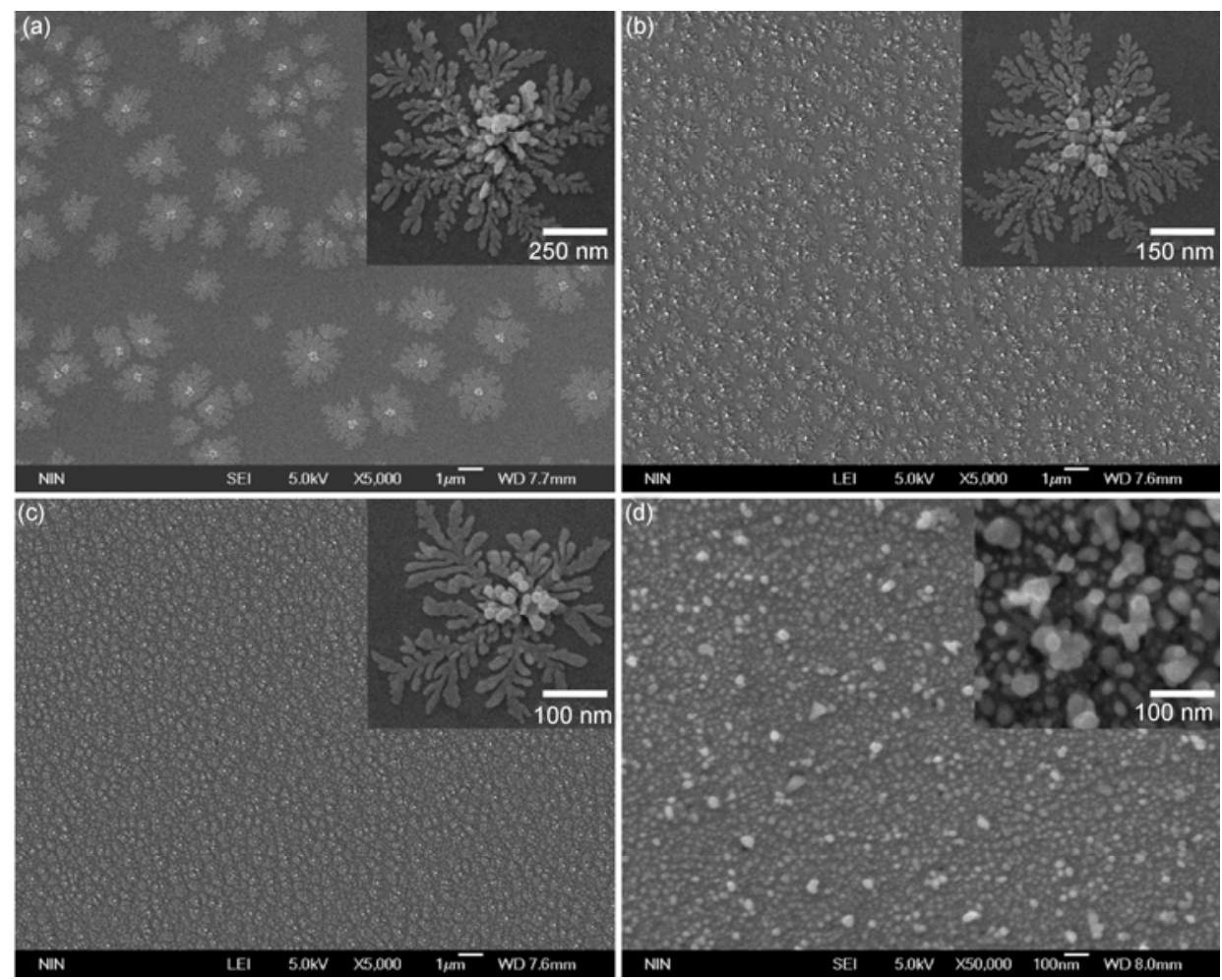

Figure 1 SEM image of electrodeposited silver.

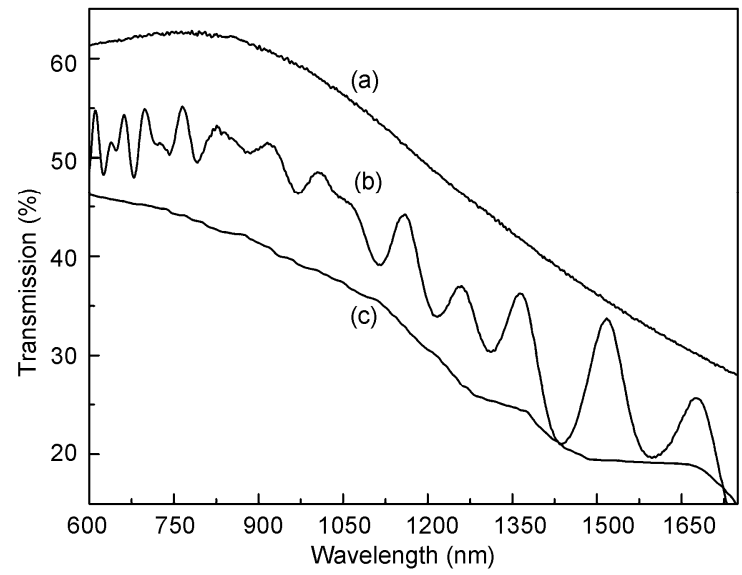

Figure 2 The transmission spectra. (a) Non-dendritic structures. (b) Dendritic cells structures. (c) Silver particles structures.

$1183 \mathrm{~nm}, 1404 \mathrm{~nm}$ and $1642 \mathrm{~nm}$, respectively, and focusing positions get farther as the increasing of the wavelength. To further validate our results, another slab focusing experiment was performed for comparison by testing two kinds of samples, I/P/I and I/f/P/f/I, using the same apparatus (Figure 3(e)). The experimental results revealed that when there are no dendritic structures (I/P/I), the light intensity is decreased slowly, as the curve $11(\lambda=829 \mathrm{~nm})$ and $12(\lambda=829 \mathrm{~nm})$ shown in Figure 3(e). When there are silver film structures $(\mathrm{I} / \mathrm{f} / \mathrm{P} / \mathrm{f} / \mathrm{I})$, light intensity decreases quickly, as shown in Figure 3(e), curve 13 ( $\lambda=709 \mathrm{~nm})$ and curve $14(\lambda=800 \mathrm{~nm})$. Both of these samples have no focusing behavior at all.

From the results of the slab focusing experiments as shown in Figure 3, we can see that the sample composed of silver dendritic structures exhibits a prominent focusing behavior at the wavelength corresponding to the maximum transmission coefficient.

\section{Fabrication and test of the tapered metama- terial optical waveguide}

\subsection{Fabrication of the tapered metamaterial optical waveguide}

The tapered waveguide is composed of two optical metamaterial samples. To observe the trapped rainbow phenomenon, a white collimated light beam with diameter around $1 \mathrm{~mm}$ is incident from the ordinary waveguide and then coupled into the tapered waveguide. We measure the spectra of transmitted light from the upper surface of LHH along the light propagation direction using fiber spectrometer (USB2000, Ocean Optics, Inc), as shown in Figure 4.

\subsection{Testing results of the optical waveguide}

The optical measurement of the waveguide was performed by using xenon lamp (XQ150) as light source, a monochromator (OMNI-300) for obtaining a desired frequency 

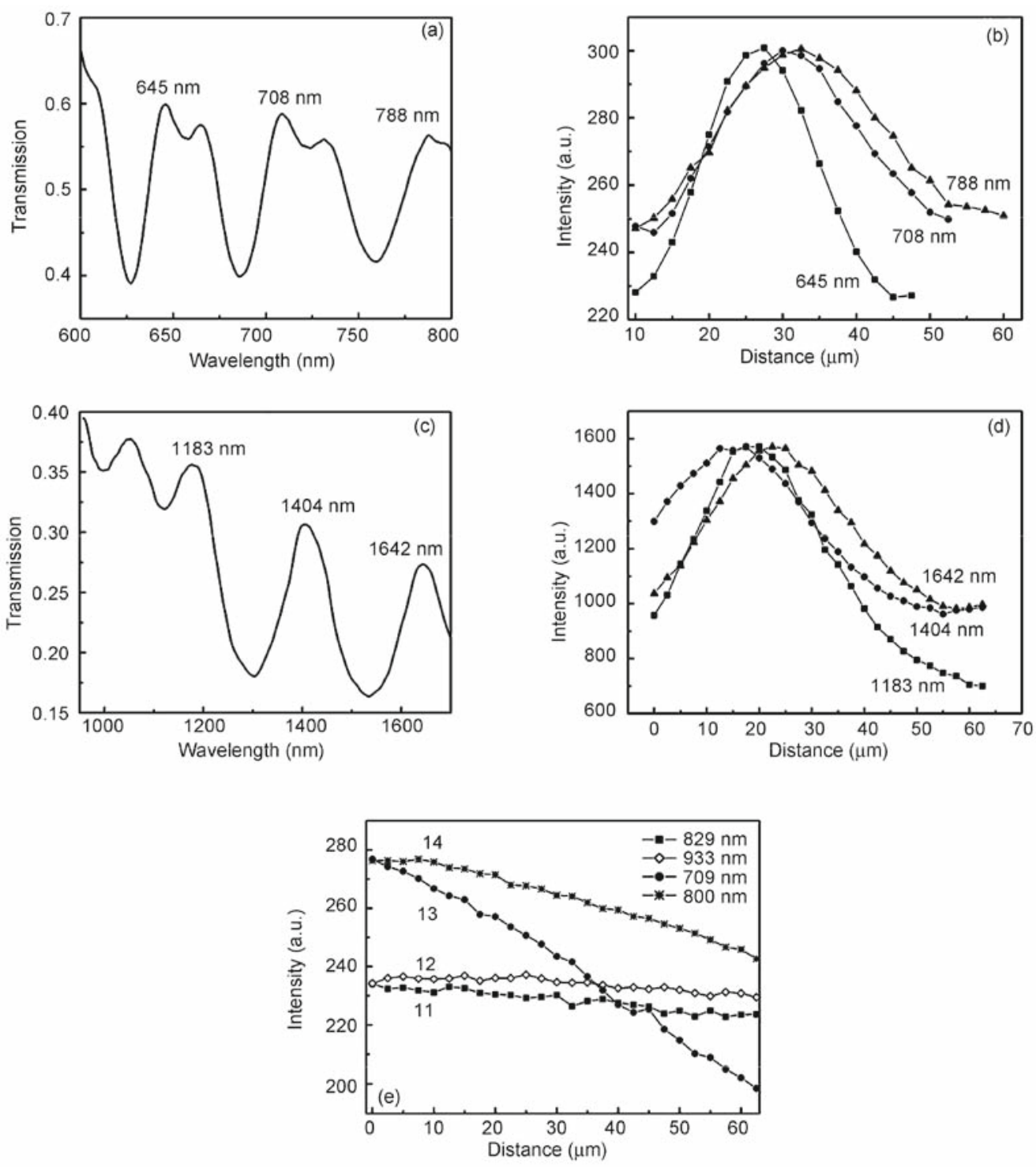

Figure 3 Slab focusing experiments. (a) and (c) The transmission spectrum of dendritic sample, corresponding to Figure 3(b) and (d), respectively.

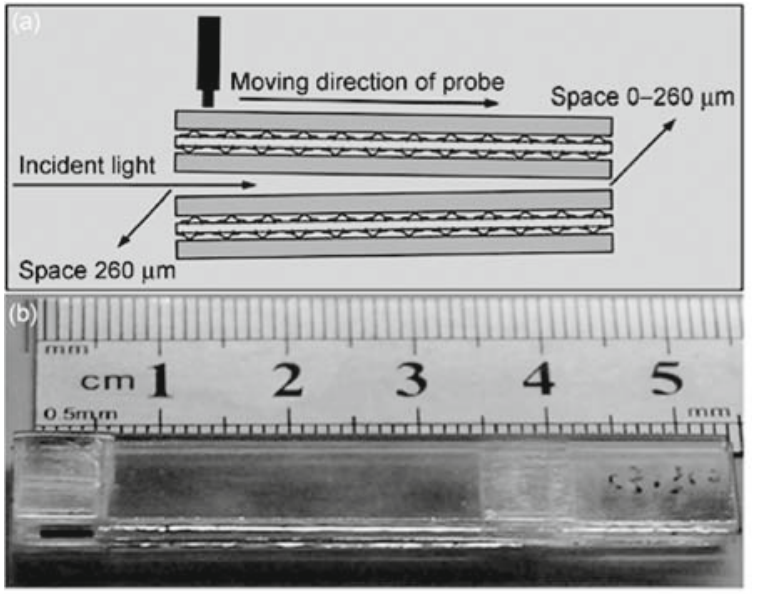

Figure 4 Scheme (a) and sample (b) of tapered optical metamaterials waveguide. component of visible light, a fiber spectrometer (USB2000) as detector fixed on a 3D micro-positioner to adjust the distance between the sample and the detector at $X$ direction and $Y$ direction, an aperture and a collimating lens. Figure 5 shows the measurement results of the waveguide (wedge angle $3.8 \times 10^{-3} \mathrm{rad}$ ). The fiber probe is moved $10 \mathrm{~mm}$ with the step $200 \mu \mathrm{m}$ during the measurement process, and the results are indicated in Figure 5(a) and (b). The results demonstrate that the peak wavelength of spectra shows an obvious red shift and the spatial separation of the spectrum appears.

The shift in peak wavelength per unit length sample is $4.1 \mathrm{~nm} / \mathrm{mm}$ as the fiber probe moves. Each frequency component of the wave packet is stopped at a different waveguide thickness, leading to the spatial separation of its spectrum. 

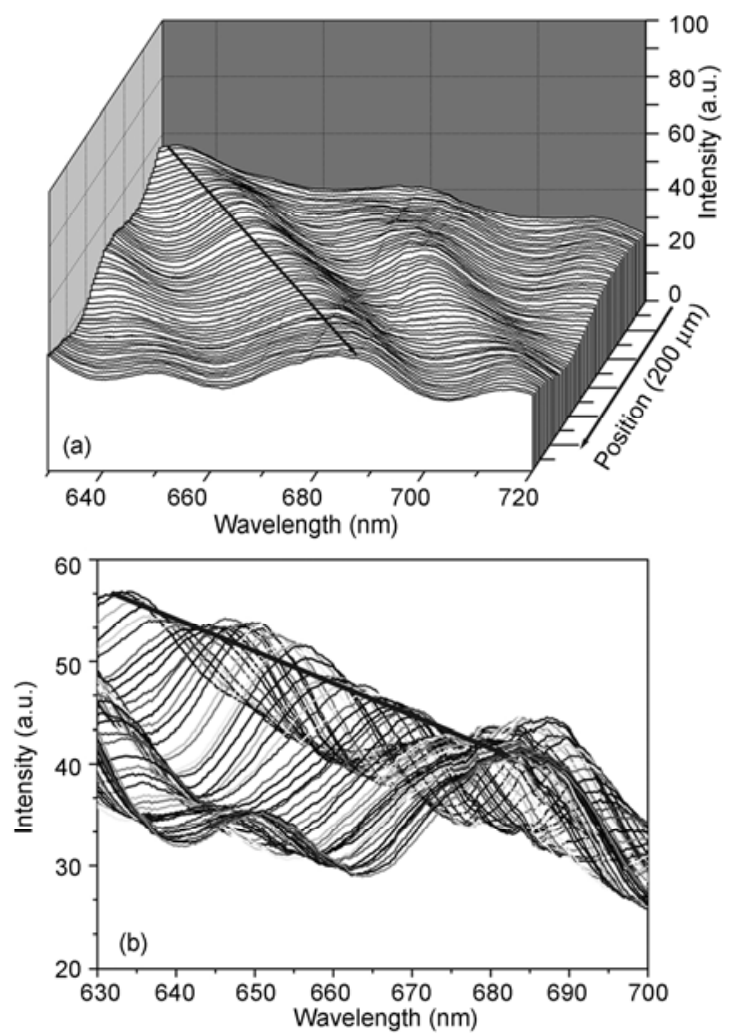

Figure 5 Spectra of transmitted light from the upper surface of tapered waveguide along the direction of the light propagation measured by fiber spectrometer. The origin of coordinate is located on the interface of ordinary waveguide and negative refractive index waveguide. (a) Spatial figure; (b) plane figure. The peak wavelengths in measured spectra exhibit a red-shift property as the tapered fiber probe moves away from the origin of the coordinate.

In order to obtain the relation between the shifts in peak wavelength per unit length sample and the wedge angle, a series of experiments were performed on the waveguides of different wedge angles. The shift in peak wavelength per unit length sample are 0, 3.8, 9.7, 12.2, 10.1, 7.2, 5.9, 2.0 and 0 $\mathrm{nm} / \mathrm{mm}$, respectively, when the wedge angles are $0,0.2,1.1$, 1.5, 1.8, 2.7, 3.1, 4.7 and 9.6 milliradian, as shown in Figure 6.

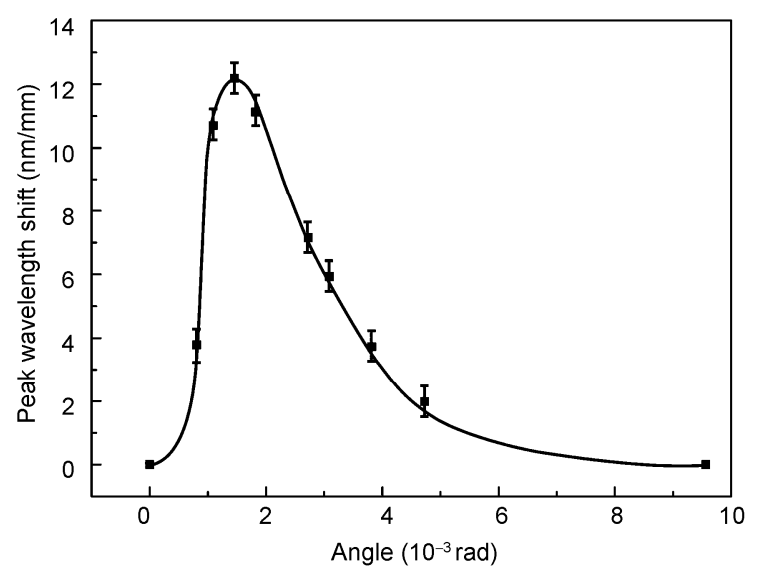

Figure 6 Fitted curve of the shift in peak wavelength per unit length sample vs. the angle formed by the two LHMs in tapered waveguide.
In Figure 6, we can see that there is no shift in the peak wavelength of the measured spectrum if the inclination of tapered waveguide is zero or exceeds a critical value (9.6 milliradian). The frequency components of wave packet separate at positions with different guide thicknesses only if the inclination of tapered LHH is greater than zero and smaller than the critical value. The spatial separation of spectrum can be effectively tuned by adjusting the inclination of tapered waveguide, which can be used for storing photons, slow-light research, quantum information processing, communication networks and signal processors.

\section{Conclusions}

In summary, a silver dendritic optical metamaterials are prepared using the electrochemical deposition method, and the transmission and focusing behaviors are investigated. The experimental results show that the samples reveal a multiple pass-bands transmission spectrum in the wavelength range from 600 to $1750 \mathrm{~nm}$ and a prominent focusing behavior at the wavelength corresponding to the maximum transmission coefficient. Two silver dendritic optical metamaterials are combined into a tapered optical waveguide, and the spectra of transmitted light at the surface of the tapered optical waveguide is measured by using the fiber spectrometer along the light propagation direction. The results demonstrate that if the wedge angle of tapered waveguide is greater than zero and smaller than the critical value, each frequency component of the wave packet is stopped at a different guide thickness, leading to the spatial separation of its spectrum. The maximum shift in peak wavelength is $12.2 \mathrm{~nm} / \mathrm{mm}$ when the wedge angle is 1.5 milliradian.

This work was supported by the National Natural Science Foundation of China (50632030 and 50872113), the National Basic Research Program of China (2004CB719805) and the Defense Basic Research Program of China.

1 Veselago V G. Electrodynamics of substances with simultaneously negative values of and magnetic permeabilities. Sov Phys Usp, 1968, 10: 509-514

2 Shelby R, Smith D R, Schulrz S. Experimental verification of a negative index of refraction. Science, 2001, 292: 77-79

3 Houck A A, Brock J B, Chuang I L. Experimental observations of a left-handed material that obeys Snell's Law. Phys Rev Lett, 2003, 90: 137401-137404

4 Parazzoli C G, Greegor R B, Li K, et al. Experimental verification and simulation of negative index of refraction using Snell's Law. Phys Rev Lett, 2003, 90: 107401-107404

5 Pendry J B. Negative refraction makes a perfect lens. Phys Rev Lett, 2000, 85: 3966-3969

6 Smith D R, Padilla W J, Vier D C, et al. Composite medium with simultaneously negative permeability and permittivity. Phys Rev Lett, 2000, 84: 4184-4187

7 Yen T J, Padilla W J, Fang N, et al. Terahertz magnetic response from artificial materials. Science, 2004, 303: 1494-1496

8 Zhang F L, Zhao Q, Liu Y H. Behaviour of hexagon split ring reso- 
nators and left-handed metamaterials. Chin Phys Lett, 2004, 21: 1330-1332

9 Zhang S, Fan W, Panoiu N C, et al. Experimental demonstration of near-infrared negative-index metamaterials. Phys Rev Lett, 2005, 95 : 137404-137407

10 Dolling G, Wegener M, Soukoulis C M, et al. Negative-index metamaterial at $780 \mathrm{~nm}$ wavelength. Opt Lett, 2007, 32: 53-55

11 Liu H, Zhao X P, Yang Y, et al. Fabrication of infrared left-handed metamaterials via double template assisted electrochemical deposition. Adv Mater, 2008, 20: 2050-2054

12 Zhou X, Fu Q H, Zhao J, et al. Negative permeability and subwavelength focusing of quasi-periodic dendritic cell metamaterials. Opt Express, 2006, 14: 7188-7197

13 Zhou X, Zhao X P. Resonant condition of unitary dendritic structure with overlapping negative permittivity and permeability. Appl Phys Lett, 2007, 91: 181908-1-3

14 Zhao X P, Zhao Q, Kang L, et al. Defect effect of split ring resonators in left-handed metamaterials. Phys Lett A, 2005, 346: 87-91

15 Zhao Q, Zhao X P, Kang L, et al. The defect effect in the one-dimensional negative permeability material. Chin J Phys, 2004, 53: 2206-2211
16 Luo C R, Kang L, Zhao Q, et al. Effect of nonuniform-defect split ring resonators on the left-handed metamaterials. Chin J Phys, 2005, 54: 1607-1612

17 Liu B Q, Zhao X P, Zhu W R, et al. Multiple pass-band optical left-handed metamaterials based on random dendritic cells. Adv Funct Mater, 2008, 18: 3523-3528

18 Tsakmakidis K L, Boardman A D, Hess O. Trapped rainbow' storage of light in metamaterials. Nature, 2007, 50: 397-401

19 Tsakmakidis K L, Klaedtke A, Aryal D P, et al. Single-mode operation in the slow-light regime using oscillatory waves in generalized left-handed heterostructures. Appl Phys Lett, 2006, 89: 201103-201105

20 Gan Q, Fu Z, Ding Y J, et al. Ultrawide-bandwidth slow-light system based on THz plasmonic graded metallic grating structures. Phys Rev Lett, 2008, 100: 256803-256806

21 Zhao X P, Luo W, Hang J X, et al. Trapped rainbow effect in visible light left-handed heterostructures. Appl Phys Lett, 2009, 95: 071111

22 Patanjali V P, Wentao T L, Plarenta V, et al. Imaging by flat lens using negative refraction. Nature, 2003, 426: 404

23 Pendry J B. Perfect cylindrical lenses. Opt Express, 2003, 11: $755-760$

Open Access This article is distributed under the terms of the Creative Commons Attribution License which permits any use, distribution, and reproduction in any medium, provided the original author(s) and source are credited. 